\title{
Fourier optics modelling of coherence scanning interferometers
}

de Groot, Peter, Colonna de Lega, Xavier, Su, Rong, Coupland, Jeremy, Leach, Richard

Peter de Groot, Xavier Colonna de Lega, Rong Su, Jeremy Coupland, Richard Leach, "Fourier optics modelling of coherence scanning interferometers," Proc. SPIE 11817, Applied Optical Metrology IV, 118170M (5 August 2021); doi: 10.1117/12.2595668

EDIE Event: SPIE Optical Engineering + Applications, 2021, San Diego, California, United States 


\title{
Fourier optics modeling of coherence scanning interferometers
}

\author{
Peter de Groot ${ }^{\mathrm{a}}$, Xavier Colonna de Lega ${ }^{\mathrm{a}}$, Rong Su${ }^{\mathrm{b}}$, Jeremy Coupland ${ }^{\mathrm{c}}$ and Richard Leach ${ }^{\mathrm{d}}$ \\ a'Zygo Corporation, Laurel Brook Road, Middlefield, CT, USA \\ bShanghai Institute of Optics and Fine Mechanics, Shanghai, China \\ ${ }^{\mathrm{c}}$ Loughborough University, Loughborough, UK \\ ${ }^{\mathrm{d}}$ University of Nottingham, Nottingham, UK
}

\begin{abstract}
We propose an instrument model for coherence scanning interferometry using familiar Fourier optics methods, the spectrum of plane waves, and the assumption that the light source spectral bandwidth is the dominant factor in determining fringe contrast as a function of optical path length. The model is straightforward to implement, is computationally efficient, and reveals many of the common error sources related to the optical filtering properties of the imaging system. We quantify the limits of applicability of the model related to the geometrical approximations for conventional Fourier optics, particularly for high numerical apertures, and when using the fringe contrast for determining surface heights. These limitations can be overcome by using a three-dimensional imaging model.
\end{abstract}

Keywords: Interferometry, interferometer, metrology, topography, CSI, Fourier optics

\section{INTRODUCTION}

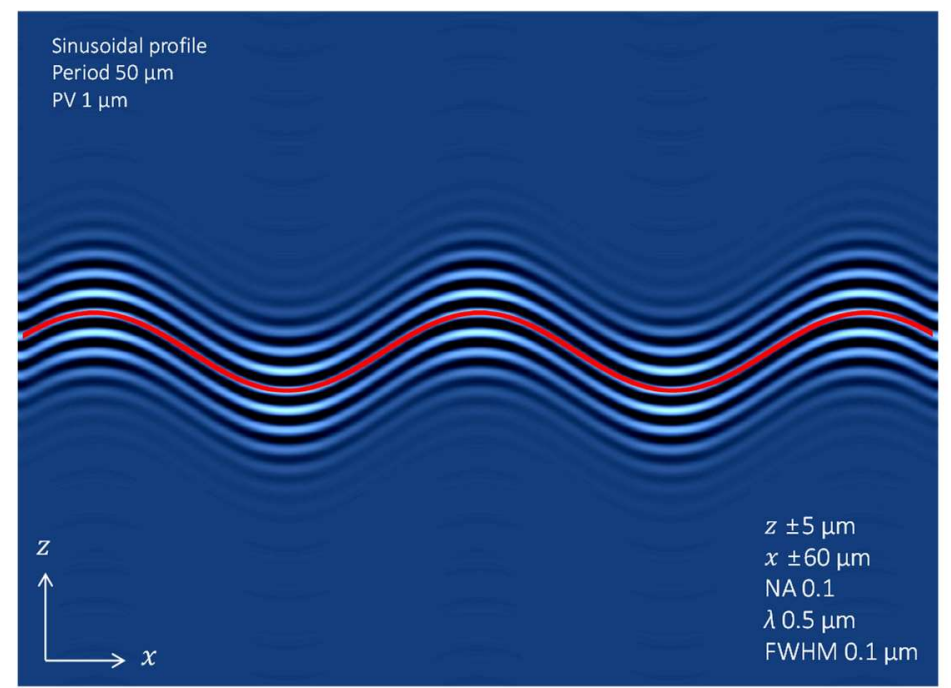

Figure 1: Simulated interference fringe signal detected by a camera for a sinusoidal surface topography when using a spectrally broadband light source, as the interference objective is scanned in the $z$ direction.

The central red line represents the object surface profile on the same scale.

The image in Figure 1 is simulated data from a coherence scanning interferometry (CSI) microscope, showing interference fringes acquired by a camera during an axial scan of the interference objective. Both the shape of the interference fringes and their variation in contrast are suggestive of the surface profile, shown as a solid red line in the figure. The image illustrates the principle of CSI while raising several questions, including how best to convert these data into a measured surface profile, and whether we can use simulations to predict the performance of CSI depending on surface topography. 
The success of CSI has motivated research into physics-based models to predict the interference signals and measurement results for these instruments [1-8]. These models address surface topographies and material characteristics that vary from simple to complicated, consistent with the wide range of applications for CSI. In this paper we propose an approximate model that builds on the Fourier optics approach for monochromatic interferometry presented at last year's SPIE conference [9]. While illustrating the basic features of a CSI system and fundamental sources of uncertainty, the model also provides a means for determining under what circumstances an approximate model of this kind generates meaningful results, and when conversely more accurate models of surface diffraction and optical filtering are recommended.

This paper is structured as follows. After a review of the principles of white-light interferometry and data processing methods, we propose the Fourier optics simulator for predicting CSI signals and measurement results. Several examples illustrate the use of the model for understanding error sources and predicting performance characteristics, such instrument response as a function of spatial frequency. This is followed by an analysis of the limitation of the model attributable to the geometrical approximations in its development. Finally, we consider approaches to more accurate modeling methods, as well as perspectives on further research in this area.

\section{INTERFERENCE MICROSCOPY}

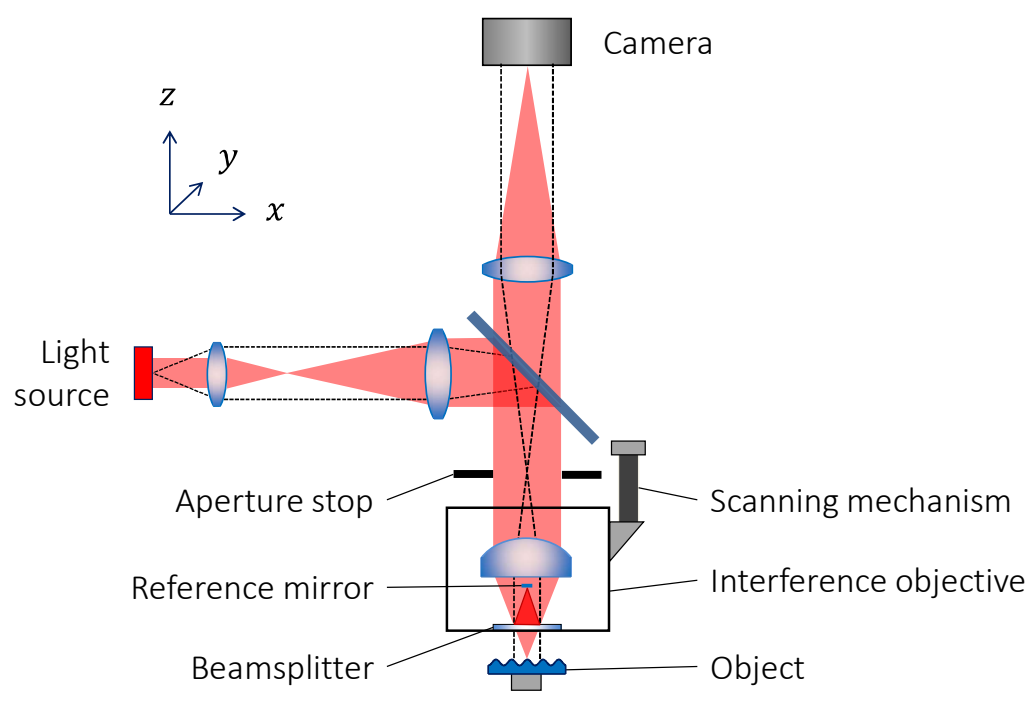

Figure 2: Model of a microscope with a Mirau type interference objective.

Figure 2 shows a common microscope configuration for measurement of surface heights using interferometry [10]. The principle of measurement, at least as a starting concept, relies on the assumption that phases are directly proportional to object surface heights:

$$
\theta_{o}(x)=2 \pi K h_{o}(x)
$$

where the subscript $o$ refers to the object, $h_{o}$ is the local surface height along the orthogonal $z$ direction, and $x$ is an abbreviation for the $x, y$ coordinates in a plane that coincides with the surface [11]. The interference fringe frequency $K$ along the $z$ axis depends on the illumination geometry as well as the wavelength $\lambda$.

A common approximation is to summarize the illumination geometry with an obliquity factor $\Omega$ that accounts for the illumination numerical aperture (NA) for the objective [12]. The fringe frequency is then 


$$
K=2 / \lambda \Omega .
$$

A value for the obliquity factor for aplanatic imaging, uniform illumination of the objective pupil is

$$
\Omega=\frac{3}{2}\left(\frac{1-\cos ^{2}(\beta)}{1-\cos ^{3}(\beta)}\right),
$$

where the maximum incident angle for the illumination cone in terms of the NA value $A_{N}$ is

$$
\beta=\sin ^{-1}\left(A_{N}\right)
$$

After measuring the phase distribution $\theta_{I}$ over the surface, for example by phase shifting interferometry (PSI), the calculated topography is

$$
h_{I}(x)=\theta_{I}(x) / 2 \pi K
$$

A known limitation when using a single wavelength $\lambda$ is that the interference phase $\theta_{I}$ can only be determined within a $2 \pi$ interval. One solution, known since the earliest days of interferometry, is to use a spectrally-broadband or "white light" source [13]. The incoherent superposition of interference patterns for multiple wavelengths results in a signal for which the interference fringe contrast reaches a maximum at the stationary phase point, corresponding to zero group-velocity path difference. The variation in fringe contrast provides a means to determine the interference fringe order, or alternatively, to determine surface heights without relying on interference phase at all [14-16].

The general concepts of white-light interferometry can be extended to any method for which the interference fringe contrast varies with surface height and objective scan position. It is common to equate CSI with white-light interferometry, and to assume for example that the width of the coherence envelope is inversely proportional to the spectral bandwidth [17]. However, it is possible to have signals similar to those shown in Figure 2 without using white light, if the light source is spatially extended and the illumination optics have an NA large enough to limit the $z$-axis range of high fringe contrast [18]. In practice, in interference microscopy, the fringe contrast effect is a combination of both the broad spectral bandwidth and the focusing effects of high NA values. For this reason, the ISO term for technologies that rely on variations in fringe contrast is called CSI rather than the more narrowly defined white light interferometry [19].

To capture and analyze CSI signals, the interference objective in Figure 2 is scanned axially, while the camera records a sequence of intensity patterns. Setting aside methods for economizing data storage, the result of the acquisition is an $x, y, z$ cube of intensity values, for which an example $x, z$ cross section is shown in Figure 1 . The usual approach to analyzing these data is to extract intensity signals $I$ as a function of scan position $z$ for each image pixel $x, y$, as illustrated in Figure 3. The resulting signal, showing interference fringes and a coherence envelope with defined characteristics such as an envelope peak, is the usual starting point for developing CSI data analysis algorithms.

A common approach to CSI data analysis is to demodulate the signal to obtain the coherence envelope. The location of the peak, centroid or other envelope characteristic is directly a measure of the surface height for the corresponding image pixel. The surface heights $h_{C}$ as a function of $x$ is often referred to as the coherence profile. By analyzing the interference fringes from the same signal, for example by using a phase-shifting algorithm near the coherence peak, we can also obtain a phase profile $h_{\theta}$ that has close to the same value as the result $h_{I}$ in Eq.(5). The coherence profile is as much as $10 \times$ 
more sensitive to additive noise and signal distortions than the phase profile, but has the important advantage of avoiding the $2 \pi$ cycle ambiguity $[10,20]$.

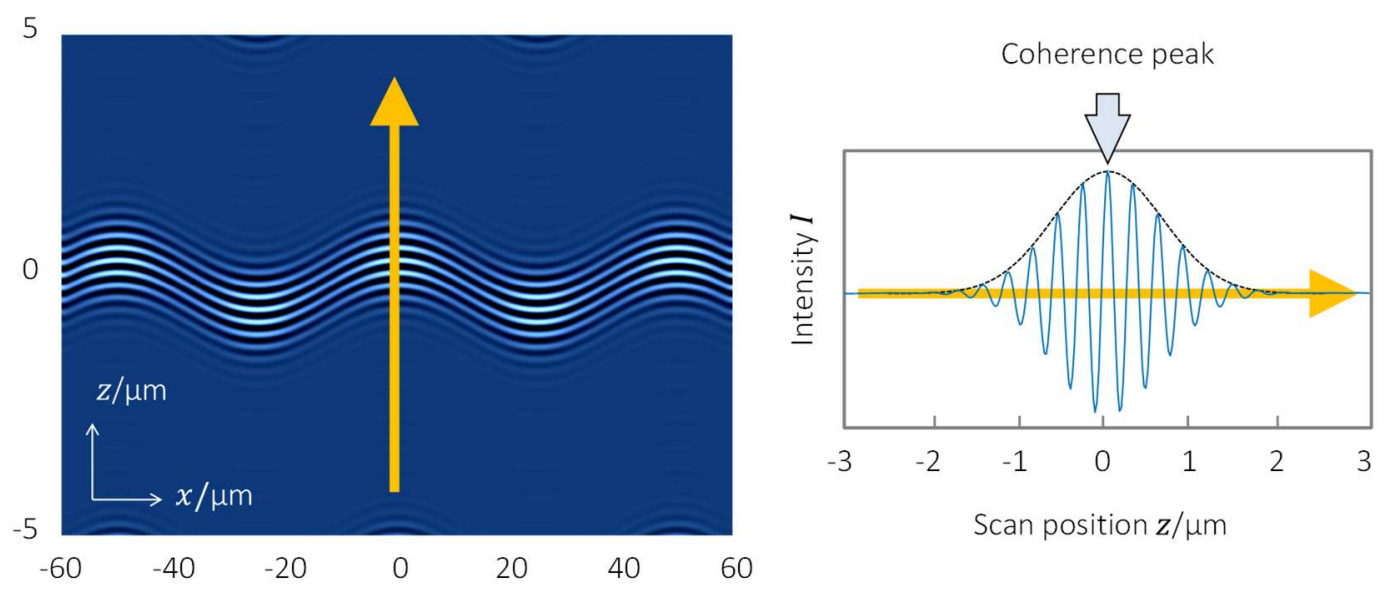

Figure 3: Extracting a CSI signal for a single image pixel.

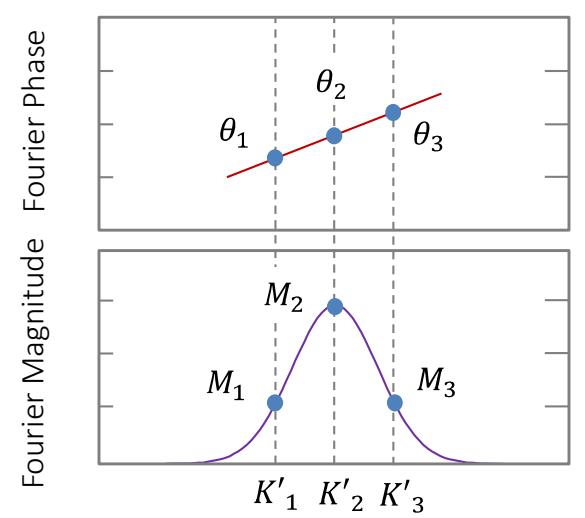

Fourier frequency

Figure 4: The FDA method of data analysis for CSI signals.

An alternative to calculating the coherence envelope and fringe phase is to transform the intensity signal $I$ into the frequency domain [21]. The Fourier transform along the $z$ direction is

$$
q\left(K^{\prime}\right)=\frac{1}{\int d z} \int I(z) \exp \left(-i 2 \pi K^{\prime} z\right) d z
$$

where the integration is over a scan range that encompasses the interference signal. These Fourier components have magnitudes and phases given by

$$
\theta=\arg \left\{q\left(K^{\prime}\right)\right\}
$$




$$
M=\left|q\left(K^{\prime}\right)\right|
$$

Figure 4 shows discretely-sampled values $\theta_{1,2,3}$ and $M_{1,2,3}$ for the Fourier transform of a signal for one image point. After removing integer multiples of $2 \pi$ between neighboring phase values, a linear fit to the phase data weighted by the Fourier magnitudes provides a phase slope and intercept for this image point. The slope of the line is a measure of the location of the signal, corresponding to the coherence profile:

$$
h_{C}(x)=\frac{1}{2 \pi} \frac{d \theta\left(x, K^{\prime}\right)}{d K^{\prime}}
$$

This result combines with the intercept $A$ at $K^{\prime}=0$ to provide a phase value at the strongest frequency $K_{M}^{\prime}$ which, in Figure 4 , is indicated by the frequency $K_{2}^{\prime}$ :

$$
\theta_{M}(x)=A(x)+2 \pi K_{M}^{\prime} h_{C}(x) .
$$

The phase translates to a phase profile using

$$
h_{\theta}(x)=\theta_{M}(x) / 2 \pi K_{M}^{\prime} .
$$

Because the coherence profile $h_{C}$ is more sensitive to sources of error than the phase profile $h_{\theta}$, it is common when measuring smooth surfaces to use the coherence profile only as a first estimate of surface height, to resolve the ambiguities in the fringe order for the phase profile $h_{\theta}[20]$.

It is important to note that the FDA method is not itself an instrument model; rather, it is one of several ways to analyze CSI signals to extract surface topography information. However, as we shall see, it is a particularly useful method in the context of modeling instrument response, as it will allow for some convenient shortcuts for signal simulations.

After this brief review of the principles of both monochromatic interference microscopy and CSI, we turn our attention to physical modeling of the interference signal and the resulting measured profiles.

\section{ELEMENTARY FOURIER OPTICS MODEL}

It is understood that the measured interference phases will be altered from the starting values by the imaging optics illustrated in Figure 2. In our 2019 SPIE paper for this conference, entitled "Does interferometry work?", it is argued that a significant potential contributor to measurement error is the spatial frequency bandwidth limits resulting from optical filtering in the imaging system [11]. This basic error mechanism can be examined, within well-defined limits, using a Fourier optics model that includes geometrical approximations to simplify the calculation.

Imaging systems are frequently characterized in optical engineering using Abbe theory and standardized concepts such as the optical transfer function (OTF) and its modulus, the modulation transfer function (MTF) [22]. These methods are most often applied to intensity objects - defined conceptually as perfectly flat surfaces having only a variation in reflectivity. If the illumination is fully incoherent, using the Fourier optics propagation of $2 \mathrm{D}$ wavefronts, the diffraction-limited imaging response is determined by linear transfer functions (TFs) in the frequency domain.

The situation is different for 3D surface topography. In principle, for such objects, there does not exist a generalized linear TF for the imaged intensity in partially coherent light, and we cannot readily apply a frequency-domain calculation using 
the OTF $[23,24]$. It can be nonetheless constructive to make several approximations that allow us to leverage familiar Fourier optics models. The first of these approximations is to replace the actual surface topography with a flat reflecting aperture function, with the local surface height represented by phase changes in the complex amplitude [25-27]. The main assumption is that the surface topography variations are small relative to the depth of field (DOF). Figure 5 illustrates this approximation, which is a familiar foundational concept in interferometry for topography measurement. Here it serves as a starting point for modeling the diffraction problem, in what we refer to as the elementary Fourier optics (EFO) method.

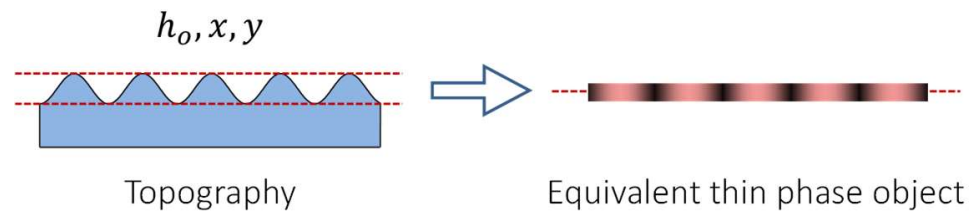

Figure 5: The thin phase object approximation, assuming that the topography variations are small relative to the DOF. The color density in the equivalent thin object represent phase variations of a complex amplitude reflectivity.

The next approximation in the EFO model for interferometers is to set aside the contribution to the measured intensity of the object surface reflection alone, given the complications of solving this problem in partially coherent light. Instead, we isolate that portion of the signal resulting from the coherent interference of the light reflected from both the object and the reference. This portion contributes the interference fringes shown in Figure 1, while the neglected portion is the background light resulting from conventional, non-interferometric imaging of the object surface and reference surfaces. This is a significant step, as it allows for modeling the interference signal generation using linear TFs.

The next approximation is not essential to the EFO method, but it provides a path to using familiar TFs such as the OTF in simulations. The most accurate way to calculate the interference signal in partially coherent light is to integrate the image contributions from all illumination incident angles, using a diffraction calculation independently for each of these contributions [28-30]. The problem is significantly simplified, with a penalty to the accuracy of the model at high NA, if we average these effects using the obliquity factor $\Omega$ defined in Eq. (3).

With this ensemble of approximations in hand, and considering for the moment just one wavelength $\lambda$, we define the following 2D complex function as a representation of the effect of the object surface on the interference fringe signal:

$$
U_{o}(x)=\exp \left[-i \theta_{o}(x)\right]
$$

where, just as in Eq. (1) and Eq. (2), we have the height-dependent phase

$$
\theta_{o}(x)=2 \pi K h_{o}(x)
$$

for the fringe frequency

$$
K=2 / \lambda \Omega \text {. }
$$

The function $U_{o}$ may be thought of conceptually as the light field immediately after reflection from the object surface, assuming uniform illumination and surface reflectivity, although it is more accurately described as the contribution of the 
object surface reflection to the interference pattern at the camera. Following conventional methods from Fourier optics [31], the light field immediately after reflection is represented by a spectrum of plane waves:

$$
\widetilde{U_{o}}\left(f_{x}\right)=\frac{1}{\int d x} \int U_{o}(x) \exp \left(-i 2 \pi f_{x} x\right) d x
$$

The tilde $(\sim)$ symbol denotes a frequency-domain representation of the corresponding space-domain quantity, and the definite integrals are over the field of view. The lateral spatial frequency $f_{x}$ for each plane wave is

$$
f_{x}=\cos \left(\alpha_{x}\right) / \lambda
$$

where $\alpha_{x}$ is the angle between the direction of the plane wave propagation and the $x$ axis. The effect on the spectrum of plane waves is a multiplication in frequency space

$$
\widetilde{U_{I}}\left(f_{x}\right)=\widetilde{O}\left(f_{x}\right) \widetilde{U_{o}}\left(f_{x}\right),
$$

where $\widetilde{O}$ is the partially-coherent TF for interferometric imaging. A familiar example TF for an interference microscope with Köhler illumination is the autocorrelation of a 2D circular pupil, which has the same form as the OTF for conventional optical imaging with a fully incoherent light source:

$$
\widetilde{O}\left(f_{x}\right)=\frac{2}{\pi}\left[\cos ^{-1}\left(\frac{\left|f_{x}\right|}{f_{\max }}\right)-\frac{\left|f_{x}\right|}{f_{\max }} \sqrt{1-\left(\frac{\left|f_{x}\right|}{f_{\max }}\right)^{2}}\right] \operatorname{rect}\left\{\frac{f_{x}}{2 f_{\max }}\right\},
$$

where the maximum detectable lateral spatial frequency is

$$
f_{\max }=2 A_{N} / \lambda
$$

For a 2D pupil, the frequency $f_{x}$ is replaced by a radius in the frequency domain. Within the implied limits of the approximations discussed above, the TF can also incorporate overall image defocus and optical aberrations [27]. Figure 6 shows example TFs illustrating the wavelength dependence of the response.

At the image plane, the coherent superposition of the filtered plane waves results in an imaged light field given by the inverse Fourier transform

$$
U_{I}(x)=\frac{1}{\int d f_{x}} \int \widetilde{U_{I}}\left(f_{x}\right) \exp \left(i 2 \pi f_{x} x\right) d f_{x}
$$

where we have reused the object-space coordinates $x, z$, equivalent to a system without distortion and with unit magnification. The interference fringe intensities are proportional to the real part of this image function

$$
I(x)=\operatorname{Re}\left[U_{I}(x)\right]
$$


and the calculation of the topography follows from a determination of the interference phase. In practice, determining the phase interferometrically may proceed by any one of a number of ways. However, for the purposes of mathematically modeling the effect of surface topography, it is often enough to calculate the phase directly from

$$
\theta_{I}(x)=-\arg \left\{U_{I}(x)\right\}
$$

The topography measurement for a single wavelength is then

$$
h_{I}(x)=\theta_{I}(x) / K
$$

and the interference signal strength is proportional to

$$
M_{I}(x)=\left|U_{I}(x)\right|
$$

This completes the description of the single-wavelength EFO model, consistent with prior work [9, 27].

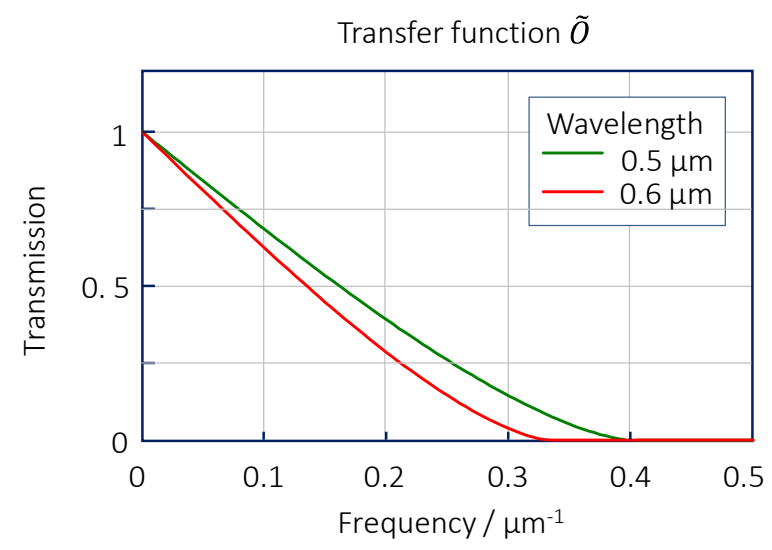

Figure 6: The imaging TF calculated from Eq. (18) for wavelengths $0.5 \mu \mathrm{m}$ and $0.6 \mu \mathrm{m}$. .

To extend the EFO model to CSI, an important decision is whether to include both optical spectrum and focus effects in the model. As has been noted in Section 2, the shape of the coherence envelope is a combination of both spectral bandwidth and illumination geometry, implying integrations over multiple incident angles and wavelengths [32]. In what follows, we will assume that the effects of the white-light spectrum are dominant. First, we define a light source spectrum that is Gaussian in wavenumber:

$$
S(k)=\exp \left[-\frac{1}{2}\left(\frac{k-k_{M}}{\sigma_{k}}\right)^{2}\right]
$$

The mean wavenumber $k_{M}$ is the inverse of the central wavelength $\lambda_{M}$ of the spectrum, and the standard deviation in wavenumbers $k$ is given by the approximately equivalent value 


$$
\sigma_{k}=\frac{1}{2 \sqrt{2 \ln (2)}} \frac{\lambda_{\Delta}}{\lambda_{M}^{2}}
$$

for a full-width at half maximum wavelength bandwidth $\lambda_{\Delta}$. In software simulations, it is often useful to normalize the spectrum $S$ so that the sum over all $k$ values is equal to unity.

In the white-light limit of low NA and with a perfectly flat part without tip and tilt, the optical spectrum may be directly associated with the Fourier frequencies using Eq.(14) and by assuming

$$
K^{\prime}=K
$$

The complex values for $U_{I}$ of the imaged light field can then be assigned to the Fourier coefficients for FDA:

$$
q(x, K)=U_{I}(x, K)
$$

The phases $\theta$ and magnitudes $M$ needed for FDA follow from Eqs.(7) and (8). It is therefore feasible in the white-light approximation to model the measured surface profile resulting from an FDA evaluation of a CSI signal, without the need to simulate the signal itself. We call this the direct to FDA method.

The direct to FDA implementation is fast and efficient, but the question remains as to how to simulate the CSI fringe image shown in Figure 1. This is useful for visualization, but also as a practical matter, for evaluating data processing methods not based on FDA. The signal for each image point follows from the inverse Fourier transform

$$
I(z)=\frac{1}{\int d K} \int q(K) \exp (-i 2 \pi K z) d K
$$

The calculation is simplified by integrating over the positive frequency $(K>0)$ the real value $q(K)$, which is equivalent to summing the interference signal for each of the wavelengths. Table 1 summarized the EFO model for CSI with white light illumination.

The number of wavelengths needed for numerical modeling of the interference signal depends on the range of possible positions of the coherence envelope in the acquired data. Defining this range as $Z$, the maximum wavelength spacing to avoid aliasing in the frequency domain is

$$
\lambda_{\Delta}=\Omega \lambda^{2} / 2 Z
$$

In practice, CSI instruments often store only that portion of the data acquisition scan that includes meaningful interference data, while recording the scan offset for each pixel in the image of the object [20,33]. This limits the range $Z$ in Eq. (6) to approximately $5 \mu \mathrm{m}$, for a maximum wavelength spacing at a mean wavelength of $0.5 \mu \mathrm{m}$ of $0.025 \mu \mathrm{m}$. For a spectral bandwidth of approximately $0.1 \mu \mathrm{m}$, it is sufficient to use three to five wavelengths near the peak Fourier magnitude for an FDA calculation of surface heights. However, for a signal simulation over a wider range, such as shown in Figure 1, many more wavelengths may be needed to fully attenuate interference signal contributions outside of the contrast envelope. 
Table 1: Summary of the EFO model for CSI, using FDA for surface profiles.

\begin{tabular}{|c|c|}
\hline Step & Reference \\
\hline For each wavelength $\lambda$ in the light source spectrum & Eq.(25) \\
\hline Calculate the corresponding fringe frequency $K$ & Eq.(2) \\
\hline Calculate object contribution $U_{o}$ to the interference effect & Eq.(12) \\
\hline Propagate through the optical system using the imaging TF $\widetilde{O}$ as a filter & Eq.(18) \\
\hline Store complex-valued result $U_{I}$ as the Fourier coefficient $q$ at this $K$ & Eq.(27) \\
\hline \multicolumn{2}{|l|}{ For each imaged pixel $x$} \\
\hline Extract the phase $\theta$ and magnitude $M$ for the Fourier coefficient $q$ & Eq.(7),(8) \\
\hline Unwrap the phase values $\theta$ as a function of $K$ & Figure 4 \\
\hline Perform a least-squares fit to the phase values $\theta$, weighted by the Fourier magnitudes $M$ & Figure 4 \\
\hline Use the phase slope for the coherence profile $h_{C}$ & Eq.(9) \\
\hline Use the phase offset and slope together from the linear fit for the phase profile $h_{\theta}$, & Eq.(10) \\
\hline \multicolumn{2}{|l|}{ Optional CSI "fringe" signal } \\
\hline \multicolumn{2}{|l|}{ For each imaged pixel $x$} \\
\hline Inverse transform the Fourier coefficients $q$ & Eq.(28) \\
\hline
\end{tabular}

\section{RESULTS}

A first example of the modeling is the sinusoidal profile shown in Figure 1. The simulated results in Figure 7 show that even in a diffraction limited system, there are small residual errors, particularly for the coherence profile. The example illustrates how a linear diffraction model can be used to predict the kind of nonlinear instrument response often encountered in practice $[8,34,35]$. The errors are the result of the bandwidth limits of the imaging TF. For the coherence profile, the larger predicted measurement errors are attributable to the wavelength dependence of TFs [11, 36]. This is one of the reasons for preferring the phase profile if the surface is sufficiently smooth.

The difference between the coherence and phase profiles is even more significant when the surface has steep slopes or sharp features that are beyond the specular NA angle limit $\beta$ of the imaging optics, defined in Eq. (4). Figure 8 shows the predicted behavior for a rectangular surface feature, illustrating the overshoots at sharp edges, often referred to as "batwings" [37]. The effect is significantly more pronounced with coherence profiles. This effect is perhaps the most frequently encountered origin of accidental $2 \pi$ errors in the phase profile, if the coherence profile is used to determine fringe order. For this reason, software for CSI commonly includes a variety of mitigation strategies, many based on a detailed analysis of the difference between the phase and coherence profiles [20]. 

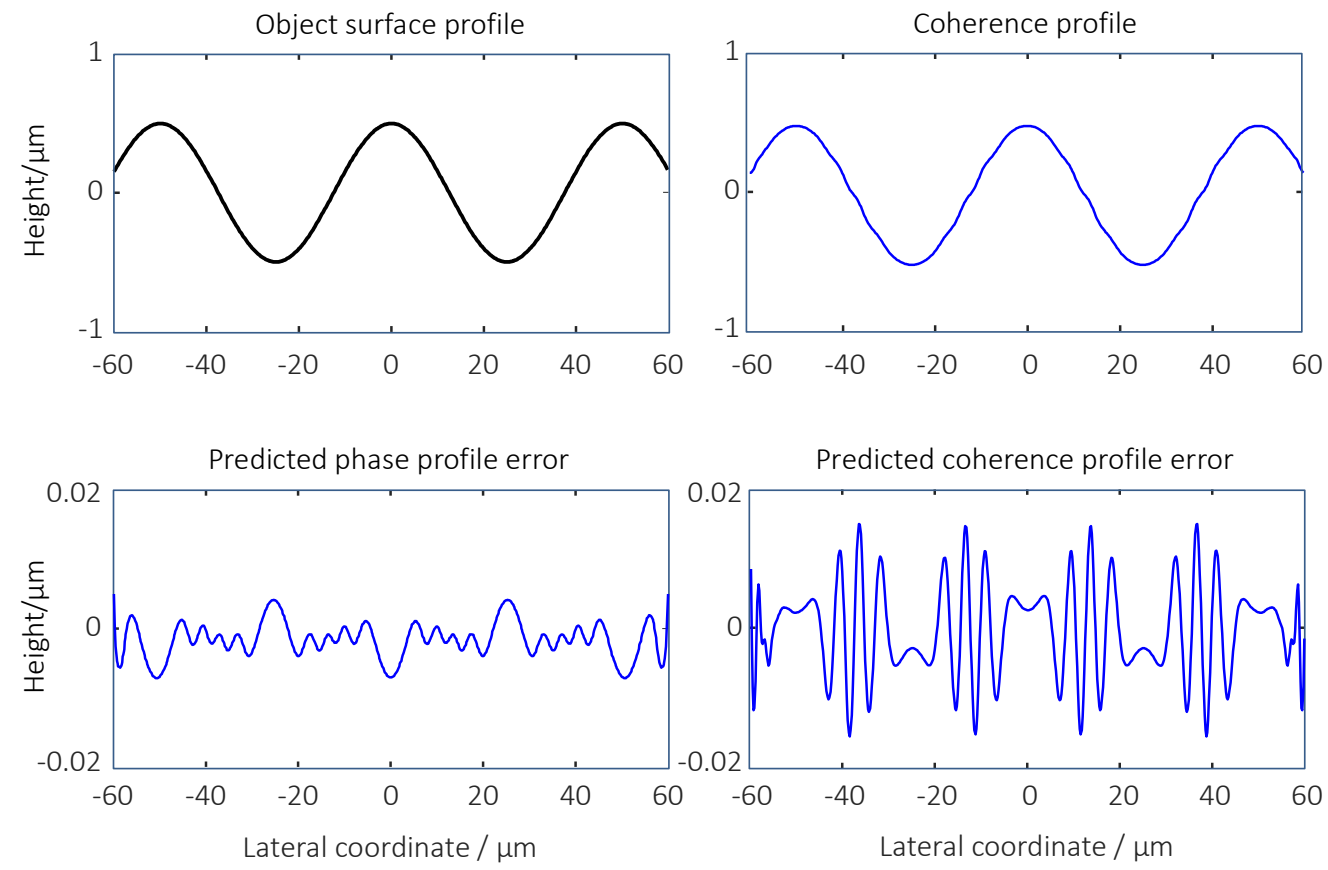

Figure 7: Simulated measurement results using the EFO model for the profile of Figure 1.

The sinusoidal period is $50 \mu \mathrm{m}$, and the peak-valley height range is $1 \mu \mathrm{m}$.

The mean wavelength is $0.5 \mu \mathrm{m}$, the FWHM bandwidth is $0.1 \mu \mathrm{m}$, and the NA is 0.1 .
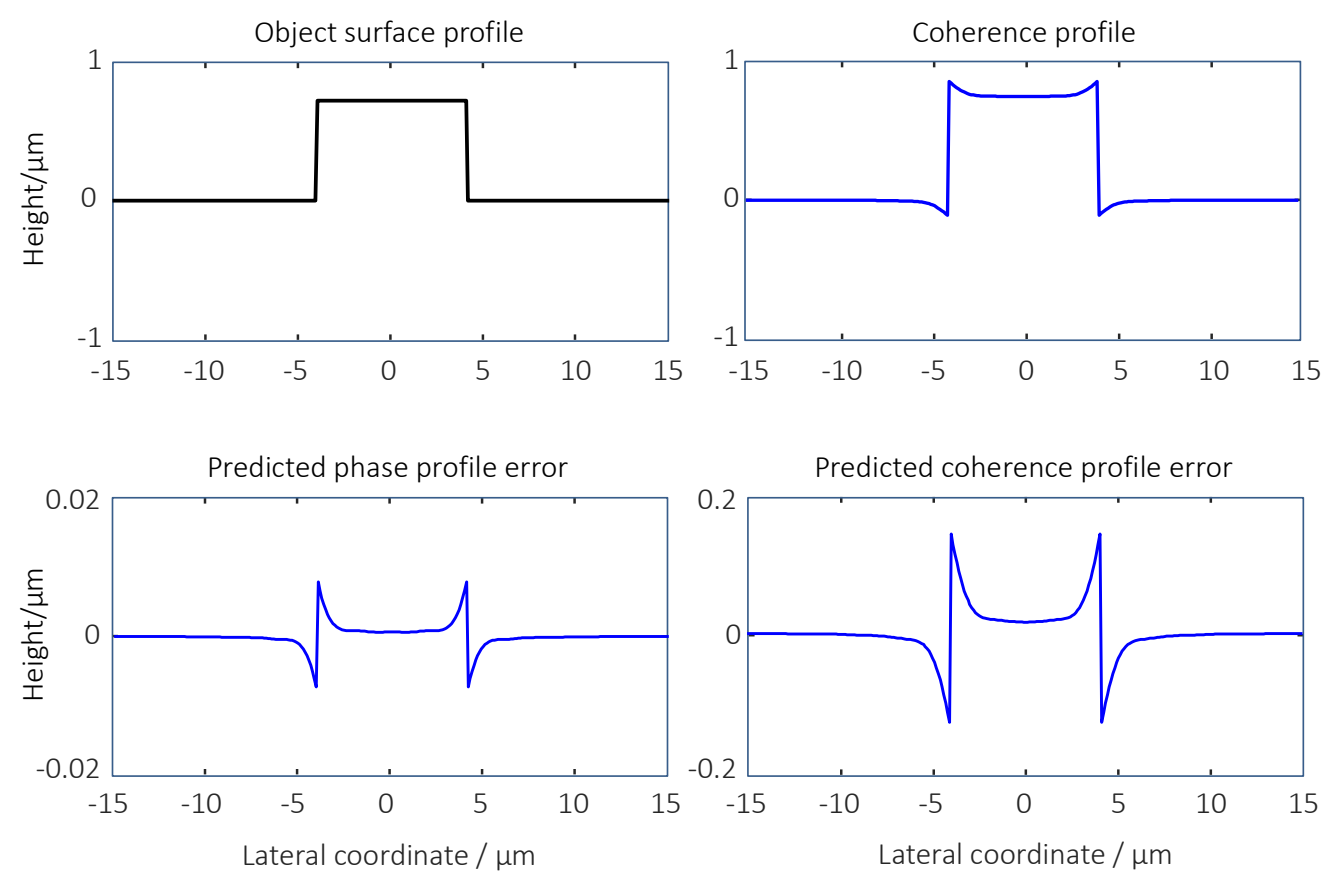

Figure 8: Simulated results using the EFO model for an $8 \mu \mathrm{m}$ wide rectangular surface feature with a height of $0.75 \mu \mathrm{m}$.

The mean wavelength is $0.57 \mu \mathrm{m}$, the FWHM bandwidth is $0.12 \mu \mathrm{m}$, and the NA is 0.15 .

Note the $10 \times$ difference in height scale for the predicted errors for the coherence and phase profiles. 

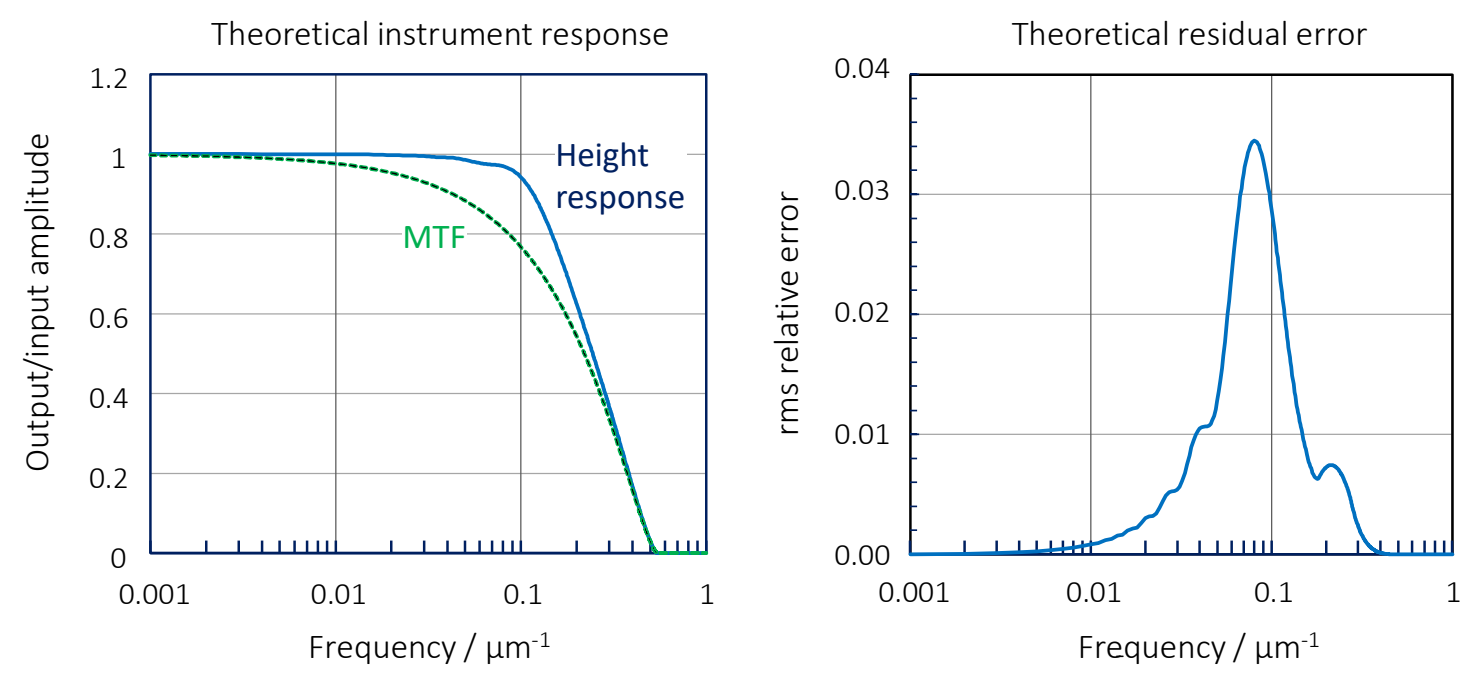

Figure 9: EFO modeling analysis of instrument response as a function of surface spatial frequency for phase profiles, for a constant maximum surface slope equal to $50 \%$ of the geometrical NA limit. The left-hand graph shows the results in comparison with the optical imaging MTF. The right-hand graph shows the rms error in the measured profile.

The mean wavelength is $0.57 \mu \mathrm{m}$ and the NA is 0.15 .

One of the uses of the EFO model is for the evaluation of instrument response as a function of spatial frequencies. This kind of analysis is useful for determining whether an instrument is suitable for measuring the power spectral density (PSD) of specific surfaces [38]. Figure 9 illustrates such an analysis for an interference microscope measuring sinusoidal surface profiles using phase information. A sequence of simulated profiles is presented to the model, with sinusoidal amplitudes scaled inversely with the spatial frequency, such that the maximum surface slope angle is at $50 \%$ of the NA angle limit $\beta$ for all frequencies. The corresponding amplitudes range from $12 \mu \mathrm{m}$ at a frequency of $0.001 \mu \mathrm{m}^{-1}$ to $0.012 \mu \mathrm{m}$ at $1 \mu \mathrm{m}^{-1}$ for an NA of 0.15. The left-hand graph in Figure 9 shows the relative measured amplitude of the sinusoidal input amplitude, while the right-hand graph in Figure 9 shows the rms nonlinearity at each frequency relative to the input amplitude.

Although not shown in Figure 9, detailed calculations for other angle limits show that for smaller surface height variations, the response curve approaches that of the MTF for conventional intensity imaging in the same optical system, and the results are almost perfectly linear [39]. Contrarily, for coherence profiles, the height response is nonlinear even for modest slopes and surface height variations, even though there are many applications involving rough surface textures that are well served by CSI measurement modes based on coherence alone.

The results shown here are representative of what is observed experimentally [20,27], and have also been confirmed by direct comparison with more advanced models [8]. However, the EFO method has its limits of validity, given the many geometrical approximations built into the model. Quantifying these limits is the topic of the next section.

\section{LIMITS OF APPLICABILITY FOR EFO MODELLING}

The approximation of a 2D complex-valued aperture function in place of the 3D topography assumes that all surface points in the topography are at the same focus position [25-27]. This gives rise to the DOF limitation for fixed-focus systems, such as PSI microscopes, noted already in Section 3. The Rayleigh formula for the DOF is

$$
D_{\text {field }}=\lambda / A_{N}^{2} .
$$


This number is one-half the focus range, but for the purpose of specifying an approximate limit for the EFO, we use $D_{\text {field }}$ as the total allowable range of surface heights. To make this more meaningful in practice, the graph in Figure 10 shows the DOF as a function of NA with several example magnifications for common interference microscope objectives. It is worth observing that typical $100 \mathrm{~mm}$ aperture laser-Fizeau interferometers have an NA of less than 0.01 . Given the limitations on resolvable fringe density, there is no practical surface height range limit for continuous surfaces below 0.1 NA for use with EFO modeling of laser Fizeau interferometers, provided that the field curvature matches that of the part to within a few millimeters. For this reason, EFO modeling is common for optical design and tolerancing [40, 41], and digital holographic refocusing is possible using 2D propagators [42].

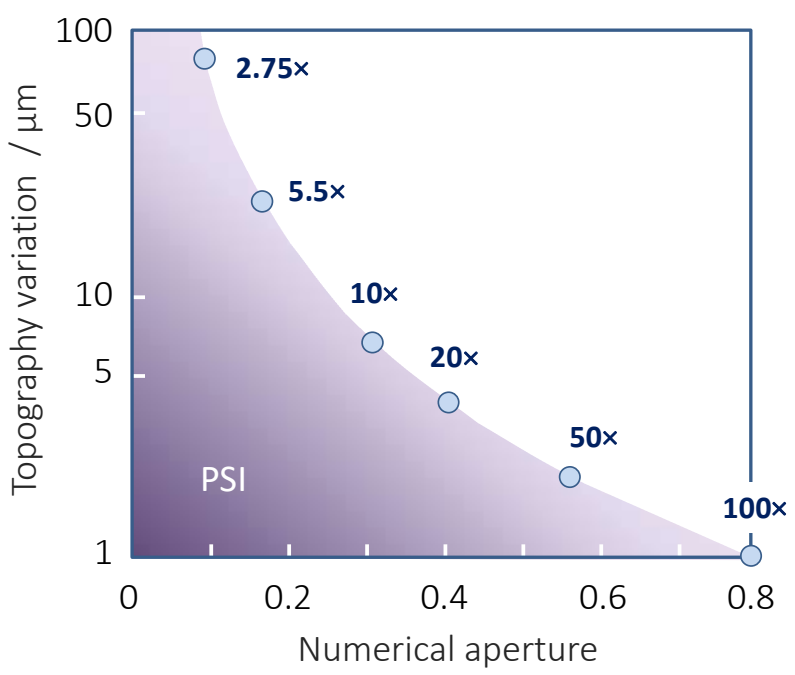

Figure 10: Limits to the allowable surface height variation for the use of the EFO model for a fixed-focus (non-scanning) system, based on the DOF. The graph includes representative magnifications for common interference objectives.

The shading qualitatively represents confidence in the modelling results, with darker shading representing greater accuracy.

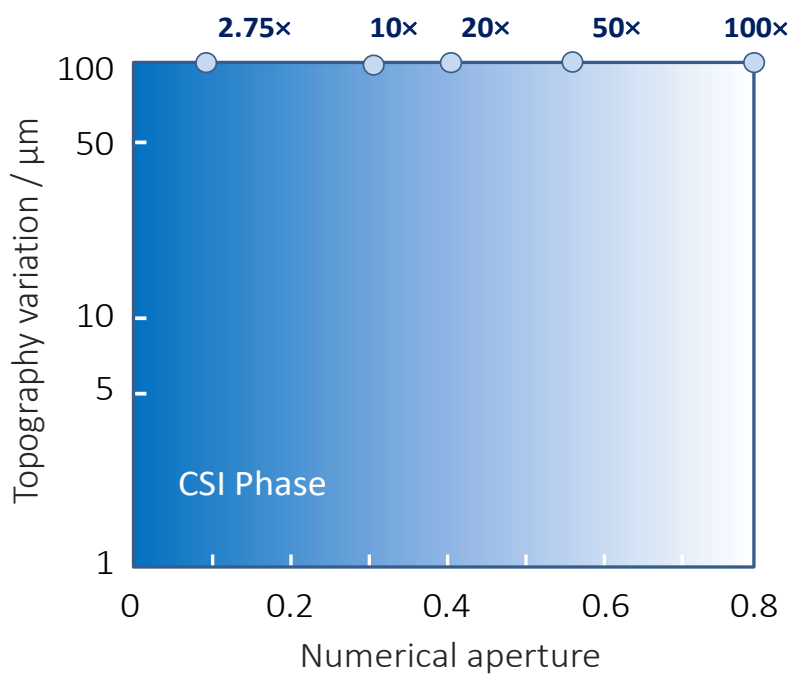

Figure 11: Limits to applicability of the EFO model for CSI when using phase information to calculate surface heights. 
For CSI, the limitations of EFO modeling are relaxed and at the same time more restricted than for fixed focus monochromatic interferometry. In most implementations of CSI, the focus position and the position of peak fringe contrast are coincident and are scanned together [10]. The measurement principle has the significant benefit that every point on the object surface is measured at the same focus position [43]. This practical benefit also means that DOF limitation for application of the EFO can be relaxed, at least for measurements based on interference phase. The CSI scan enables a piecewise evaluation across the field of view, equivalent to collapsing the entire surface topography into the thin complexvalued aperture function of the EFO model, even if the surface height range is much larger than the DOF. We have verified this phenomenon by direct comparison with more advanced 3D modeling that we will describe in Section 6. Figure 11 summarizes this finding for phase profiles in CSI, with applicability for the model over all height ranges and NA values. The progressively lighter shading is a qualitative representation of declining confidence in the result at higher NA values, given polarization-dependent material effects at large incident angles, and the use of scalar diffraction theory.

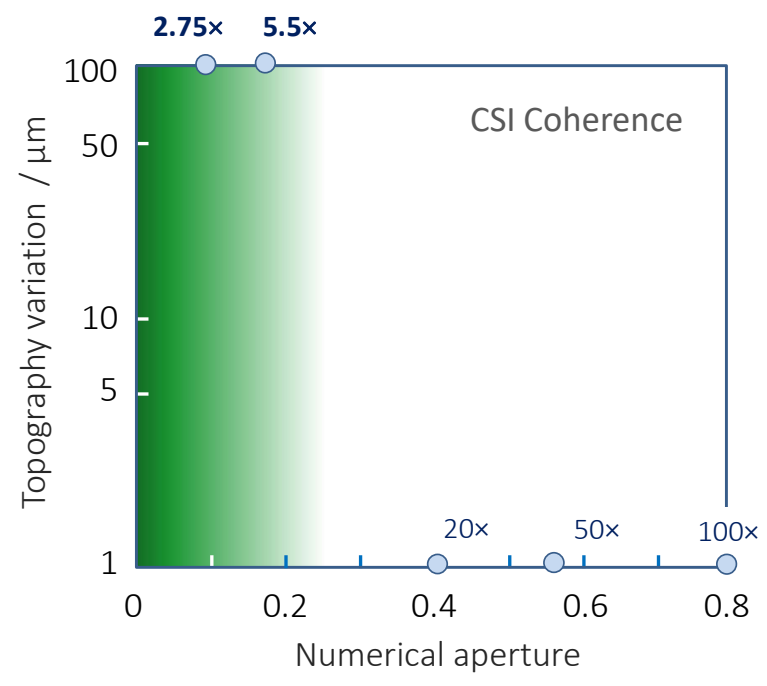

Figure 12: Limits to applicability of the EFO model for CSI for coherence profiles using common light sources. The "white light" approximation is limited to low NA values characteristic of objective magnifications below $10 \times$.

The situation is less encouraging for measurements using coherence. The white-light EFO model for CSI described in this paper assumes that the coherence envelope is shaped uniquely by the broad spectral bandwidth of the light source; whereas it is well known that at high NA values, the envelope changes in width and overall shape as a consequence of the range of illumination angles $[18,32,44]$. Consequently, a constraint for the extended height range for simple EFO modeling in CSI is that the coherence length of the illumination should be much less than the depth of focus $D_{\text {field }}$. A comparison of the EFO model presented here with more advanced methods with fewer geometrical approximations shows that "much less" is approximately a factor of five if the intent is to accurately predict residual measurement errors in the coherence profile. This requirement can be written as

$$
\sigma_{C}<D_{\text {field }} / 5
$$

where the coherence length is

$$
\sigma_{C}=1 / \sigma_{k}
$$


As a practical example, assume the coherence length is $2 \mu \mathrm{m}$ for a $0.57 \mu \mathrm{m}$ light source with a $0.12 \mu \mathrm{m}$ FWHM spectral bandwidth, and scales inversely with the bandwidth. Figure 12 summarizes this limit, where the shading once again is indicative of declining confidence in the result with increasing NA and corresponding objective magnifications.

Importantly, the limitations on NA for simulating coherence profiles in CSI are not relaxed in the limit of small surface height variations, as they are for fixed-focus measurements based on interference phase. In essence, above a certain illumination NA value, EFO modeling is simply not suited to the task of simulating the complete CSI signal well enough to predict the coherence profile results, regardless of the range of surface height variations.

The limitations of the EFO model for CSI at high NA can be overcome by abandoning the obliquity factor approximation and considering the diffracted wavefronts as a function of incident angle, using pupil-plane integration [30]. A limit case for this method is the modeling of a monochromatic, high-NA system, for which the Fourier coefficients in the FDA method map to directional cosines, rather than spectral wavenumbers [18]. However, to account for both spectral bandwidth and measurement geometry correctly, the most accurate method is to integrate over the pupil plane the diffracted plane waves for each incident plane wave in the illumination [27]. Although this is a useful approach and can be applied to nonlinear diffraction problems such as optically-unresolved surface features [45], clearly the simplicity and ease of implementation of the EFO model is lost, and it becomes compelling to start fresh with a more realistic 3D imaging solution.

\section{3D MODELS}

Conventional Fourier optics methods are based on 2D TFs along the lateral spatial $f_{x}, f_{y}$ frequency axes, with propagation and modification of wavefronts expressed in terms of planar representations of light fields. Models based on 3D TFs in $f_{x}, f_{y}$ and $f_{z}$ are common in microscopy [46], particularly confocal microscopy and high lateral resolution systems [47]. The formalism for 3D TFs can be equally well applied to holography and interferometry [48] and is of interest in any highNA system for which focus effects strongly influence the outcome of a measurement, including CSI [49-51].

Linear 3D models have a rigorous foundation in diffraction theory [47, 50]; however, for our present purpose, we consider just the geometrical aspects of 3D TFs. Figure 13 shows the $f_{x}, f_{z}$ cross-section of a 3D TF for spatially-incoherent, monochromatic light in an interference microscope. The key difference of this figure with respect to the more familiar representation shown in Figure 6 is the addition of the vertical axis $f_{z}$ to the lateral $f_{x}$ axis, with the consequence that the TF magnitude is now represented by a color map. The 2D TF shown in Figure 6 is equivalent to integrating the image shown in Figure 13 along the $f_{z}$ direction for each position along the $f_{x}$ axis.

The construction of Figure 13 starts by defining incident wavevectors $\mathbf{k}_{\mathbf{i}}$ and scattered wavevectors $\mathbf{k}_{\mathbf{s}}$. These wavevectors have a length given by the spectral wavenumber $k=1 / \lambda$, and are oriented according to their incident and scattered angles. The range of incident angles for $\mathbf{k}_{\mathrm{i}}$ sweeps out an arc $a_{i}$ consistent with the geometry of the illuminating light cone for a system having a pupil filled with spatially incoherent light. For each of these incident wavevectors $\mathbf{k}_{\mathbf{i}}$, there is an arc $a_{s}$ of scattered wavevectors $\mathbf{k}_{\mathrm{s}}$ limited by the acceptance cone of the imaging system. The combination of these two arcs defines the range of possible $f_{x}, f_{z}$ values that are detectable using interferometry. By sweeping both the $\mathbf{k}_{\mathrm{i}}$ and $\mathbf{k}_{\mathrm{s}}$ vectors through all possible angles, we obtain the 3D TF, with a magnitude distribution proportional to the number of ways in which these vectors can be oriented to reach the same $f_{x}, f_{z}$ point in frequency space.

The 3D TF allows us to predict the frequency content of a signal, such as the one shown in Figure 1, for an object surface modeled as a thin "foil" or point cloud of scattering points in $x, y, z \quad[25,52]$. The 3D TF can be measured experimentally 
by realization of the 3D point spread function using a small sphere, which scatters light in every direction [49]. The resulting detected signal will have a frequency content close to that of the 3D-TF. This method has been used to calibrate interferometers and to correct for defocus, aberrations, and tilt-dependent errors [53-55].

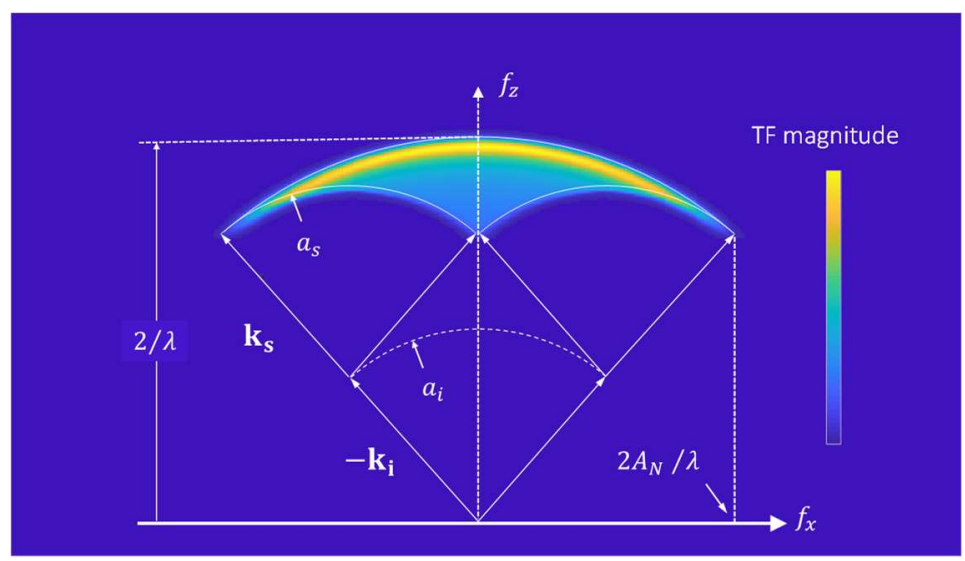

Figure 13: Geometrical construction of the cross section of a 3D-TF in monochromatic light with a linear pupil.

An approach to understanding the 3D TF is to observe its frequency limits. Along the lateral $f_{x}$ axis, the boundaries are given by the Abbe frequency, exactly as in Eq. (19). Along the vertical $f_{z}$ axis, we have a range of possible interference fringe frequencies, with the maximum possible frequency for normal incidence and specular reflection of $2 / \lambda$. For every other frequency within the boundaries of the $3 \mathrm{D} \mathrm{TF}$, there are differing fringe frequency contributions along the $f_{z}$ axis and lateral spatial frequencies along the $f_{x}$ axis. For white-light illumination, the superposition of wavevector lengths of differing lengths blurs the TF and extends its boundaries along the $f_{z}$ direction, without departing from the overall "umbrella" shape shown in Figure 13.
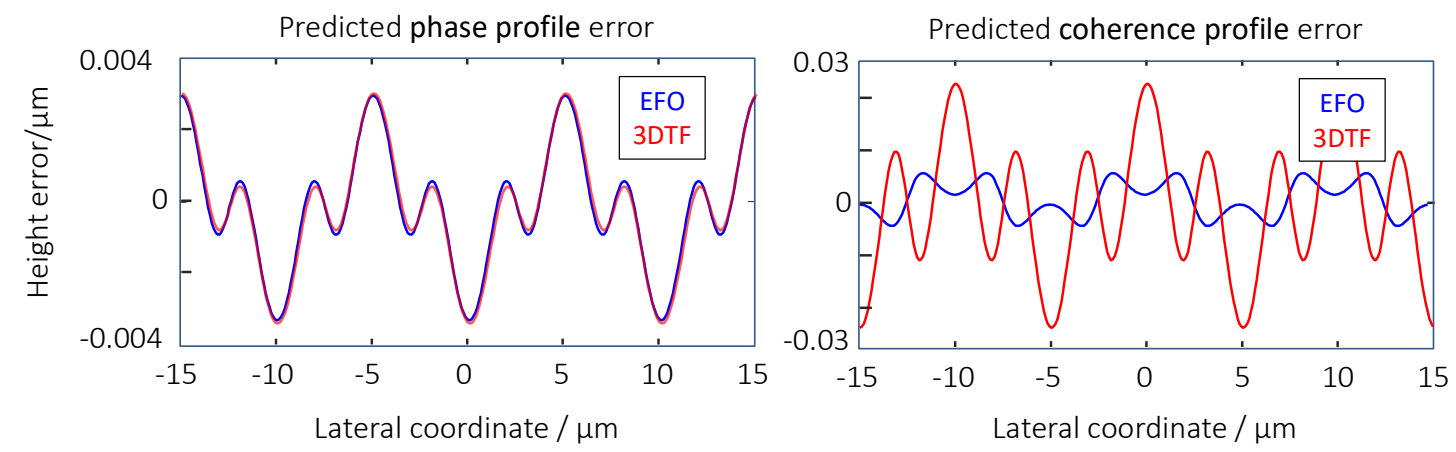

Figure 14: Simulations at NA $=0.55(50 \times$ objective $)$ for a sinusoidal profile with an amplitude of $0.15 \mu \mathrm{m}$. The mean wavelength is $0.57 \mu \mathrm{m}$ and the FWHM bandwidth is $0.08 \mu \mathrm{m}$. The predicted measurement errors for the phase profile are shown in the left-hand graph, while the predicted errors in the coherence profile are in the right-hand graph.

In the simplest EFO modeling, the use of the obliquity factor $\Omega$ defined by Eq. (2) and Eq. (3) is equivalent to averaging the frequency projections along the vertical $f_{z}$ axis, so that we can use linear 2D TFs. In this approximation, we lose all of the detail of the 3D TF regarding the variation in possible $f_{z}$ values as a function of $f_{x}$. At low NA values, this is not serious: in the limit of very small NA, the cross-section of the 3D TF would appear as a broad line parallel to the lateral 
$f_{x}$ axis shown in Figure 13, with a magnitude distribution given by the TF shown in Figure 6. However, at high NA values, the shape of the 3D TF is not well represented by this obliquity factor approximation.

The practical difference in signal simulation between 2D and 3D TFs at high NA is illustrated in the example shown in Figure 14, which shows the predicted measurement error for a sinusoidal object profile having an amplitude of $0.15 \mu \mathrm{m}$ and a period of $10 \mu \mathrm{m}$, for a $50 \times$ interference objective and diffraction-limited optics. For the phase profile, the approximations in EFO modeling with an obliquity factor are acceptable; whereas for the coherence profile, which is much more dependent on the signal shape rather than its mean phase, the EFO model underestimates the error. This is consistent with the limits of applicability described in Section 5, which have been verified by comparison with a virtual CSI model based on 3D TFs [8].

\section{SUMMARY AND FUTURE WORK}

Modern interference microscopes for surface topography measurement often employ coherence scanning methods with spectrally broadband light sources to extend the measurement range beyond one interference phase cycle. Within welldefined limits, a Fourier optics model, using familiar concepts from conventional imaging systems analysis, can predict the interference signals and measured surface profiles for these instruments. A defining simplification is the modeling of the surface topography as a complex-valued reflecting aperture function for which the surface heights are encoded as phase shifts - a common concept for topography measurement using interferometry. With this approximation in hand, we propose the EFO model summarized by Table 1 for CSI signals and measurement results.

Practical uses for EFO models include evaluating instrument response for the specification of lateral resolution and frequency response [39]. Using the EFO model presented here, we also find that errors in CSI microscopy encountered in practice can often be attributed to the wavelength dependence of the imaging transfer function, even for diffraction-limited optical systems, particularly when using the fringe contrast envelope to measure surface heights. Software developments since the discovery of CSI three decades ago target these error sources; but it is nonetheless useful to predict their magnitude using simplified data processing, to configure measurement to minimize their influence.

We find that the instrument response when using interference phase is adequately represented by the EFO model for the most commonly-used interference objectives; however, there are limitations to the accuracy of the model at higher NA values, especially when using the fringe contrast to measure surface heights. In all cases we find that a full 3D model is more reliable because it has fewer geometrical approximations, with the greatest benefit at NA values above 0.2.

The several additional simplifying assumptions of both 2D and 3D modeling as presented here, including scalar diffraction, optically smooth surfaces (relative to wavelength), neglect of material and polarization effects, and the absence of thin partially-transparent surface films, provide opportunities for further work. These developments help with obtaining the most realistic results from existing instruments, while generating ideas for new methods and techniques for optical noncontact areal surface topography measurements.

\section{ACKNOWLEDGEMENTS}

This project has received partial funding from the EMPIR program (20IND07) co-financed by the Participating States and from the European Union's Horizon 2020 research and innovation program. 


\section{REFERENCES}

[1] Xie, W., "Transfer Characteristics of White Light Interferometers and Confocal Microscopes",PhD Thesis, University of Kassel (2017).

[2] Thomas, M., Su, R., Nikolaev, N., Coupland, J., and Leach, R. K., "Modeling of interference microscopy beyond the linear regime," Optical Engineering 59(3), 034110 (2020).

[3] Coupland, J., and Nikolaev, N., "Surface scattering and the 3D transfer characteristics of optical profilers", SPIE, EPE (2020).

[4] Pineda, J., Altamar-Mercado, H., Romero, L., and Marrugo, A., "Toward the generation of reproducible synthetic surface data in optical metrology," Proc. SPIE 11397 (2020).

[5] Bischoff, J., Pahl, T., Lehmann, P., and Manske, E., "Model-based dimensional optical metrology," Proc. SPIE 11352, 113520P,1-113520P.13 (2020).

[6] Abdulhalim, I., "Spatial and temporal coherence effects in interference microscopy and full-field optical coherence tomography," Annalen der Physik 524(12), 787-804 (2012).

[7] de Groot, P. J., and Colonna de Lega, X., "Signal modeling for modern interference microscopes," Proc. SPIE 5457, 26-34 (2004).

[8] Su, R., and Leach, R. K., "Physics-based virtual coherence scanning interferometer for surface measurement," Light: Advanced Manufacturing 2(LAM2020100030), 1 (2021).

[9] de Groot, P., and Colonna de Lega, X., "Fourier optics modelling of instrument response for interference microscopy," Proc. SPIE 11490, 114900T.1-12 (2020).

[10] de Groot, P., "Principles of interference microscopy for the measurement of surface topography," Advances in Optics and Photonics 7(1), 1-65 (2015).

[11] de Groot, P., Colonna de Lega, X., Su, R., and Leach, R. K., "Does interferometry work? A critical look at the foundations of interferometric surface topography measurement," Proc. SPIE 11102, 111020G.1 - 111020G-11 (2019).

[12] Sheppard, C. J. R., and Larkin, K. G., "Effect of numerical aperture on interference fringe spacing," Applied Optics 34(22), 4731 (1995).

[13] Michelson, A. A., "Comparison of the international metre with the wavelength of the light of cadmium," Astronomy and Astro-Physics 12, 556-560 (1893).

[14] Balasubramanian, N., "Optical system for surface topography measurement," US Patent 4,340,306, (1982).

[15] Su, R. "Coherence scanning interferometry," [Advances in Optical Surface Texture Metrology], Leach, R. K., Ed., IOP Publishing, (2020).

[16] Dresel, T., Häusler, G., and Venzke, H., "Three-dimensional sensing of rough surfaces by coherence radar," Applied Optics 31(7), 919-925 (1992).

[17] Schmit, J. "White Light Interferometry," [Encyclopedia of Modern Optics], Guenther, R. D., Ed., Elsevier, Oxford (2005).

[18] de Groot, P., and Colonna de Lega, X., "Angle-resolved three-dimensional analysis of surface films by coherence scanning interferometry," Optics Letters 32(12), 1638 (2007).

[19] ISO, "25178-604:2013, Geometrical product specification (GPS) - Surface texture: Areal - Nominal characteristics of non-contact (coherence scanning interferometric microscopy) instruments," International Organization for Standardization, Geneva (2013).

[20] de Groot, P., Colonna de Lega, X., Kramer, J., and Turzhitsky, M., "Determination of fringe order in white-light interference microscopy," Applied Optics 41(22), 4571-4578 (2002).

[21] de Groot, P., and Deck, L., "Surface Profiling by Analysis of White-light Interferograms in the Spatial Frequency Domain," Journal of Modern Optics 42(2), 389-401 (1995).

[22] Boreman, G. D., [Modulation Transfer Function in Optical and Electro-Optical Systems], SPIE Press, Bellingham (2001).

[23] Ichioka, Y., and Suzuki, T., "Image of a periodic complex object in an optical system under partially coherent illumination," Journal of the Optical Society of America 66(9), 921-932 (1976).

[24] De, M., and Mondal, P. K., "Phase and Amplitude Contrast Microscopy in Partially Coherent Light," Journal of Research of the National Bureau of Standards-C. Engineering and Instrumentation 69C(3), (1965).

[25] Sheppard, C. J. R., "Imaging of random surfaces and inverse scattering in the Kirchoff approximation," Waves in Random Media 8(1), 53-66 (1998). 
[26] Frieden, B. R., "Optical Transfer of the Three-Dimensional Object," Journal of the Optical Society of America 57(1), 56-66 (1967).

[27] de Groot, P., and Colonna de Lega, X., "Fourier optics modeling of interference microscopes," J. Opt. Soc. Am. A 37(9), B1-B10 (2020).

[28] Hopkins, H. H., "Applications of coherence theory in microscopy and interferometry," Journal of the Optical Society of America 47(6), 508-526 (1957).

[29] Voelz, D. G., [Computational Fourier Optics: A MATLAB Tutorial], SPIE Press, Bellingham, WA (2011).

[30] Totzeck, M., "Numerical simulation of high-NA quantitative polarization microscopy and corresponding nearfields," Optik - International Journal for Light and Electron Optics 112(9), 399-406 (2001).

[31] Goodman, J. W., [Introduction to Fourier Optics], W. H. Freeman Macmillan Learning, New York (2017).

[32] de Groot, P., and Colonna de Lega, X., "Signal modeling for low-coherence height-scanning interference microscopy," Applied Optics 43(25), 4821 (2004).

[33] Deck, L., and de Groot, P., "High-speed non-contact profiler based on scanning white light interferometry," International Journal of Machine Tools and Manufacture 35(2), 147-150 (1995).

[34] Rhee, H.-G., Vorburger, T. V., Lee, J. W., and Fu, J., "Discrepancies between roughness measurements obtained with phase-shifting and white-light interferometry," Applied Optics 44(28), 5919-5927 (2005).

[35] Gao, F., Leach, R. K., Petzing, J., and Coupland, J. M., "Surface measurement errors using commercial scanning white light interferometers," Measurement Science and Technology 19(1), 015303 (2008).

[36] Lehmann, P., and Niehues, J. "Limitations and optimization of low-coherence Interferometry for high precision microscopic form measurement," [Fringe 2009: The 6th International Workshop on Advanced Optical Metrology], Osten, W. and Kujawinska, M., Ed., Springer Berlin Heidelberg, 41 (2009).

[37] Harasaki, A., Schmit, J., and Wyant, J. C., "Improved vertical-scanning interferometry," Applied Optics 39(13), 2107-2115 (2000).

[38] ISO, "10110-8:2010, Optics and Photonics - Preparation of drawings for optical elements and systems," International Organization for Standardization, Geneva (2010).

[39] de Groot, P., "The instrument transfer function for optical measurements of surface topography," Journal of Physics: Photonics, (2021).

[40] Wang, Q., "Fourier analysis of phase-shifting algorithms for amplitude measurement of interference fringe," Applied Optics 56(15), 4353-4357 (2017).

[41] Millstone, D., Dubin, M., and Ruiz, E., "Amplitude edge response characterization of interferometer ITF and the effect of defocus," Proc. SPIE 11490, 114900V.1-11 (2020).

[42] Deck, L. L., "Method and apparatus for optimizing the optical performance of interferometers," US Patent 10267617, (2019).

[43] Chakmakjian, S., Biegen, J. F., and de Groot, P., "Simultaneous focus and coherence scanning in interference microscopy," IWI Proceedings, 171-172 (1996).

[44] Lehmann, P., Tereschenko, S., Allendorf, B., Hagemeier, S., and Hüser, L., "Spectral composition of lowcoherence interferograms at high numerical apertures," Journal of the European Optical Society-Rapid Publications 15(1), 5 (2019).

[45] de Groot, P., Colonna de Lega, X., Liesener, J., and Darwin, M., "Metrology of optically-unresolved features using interferometric surface profiling and RCWA modeling," Optics Express 16(6), 3970 (2008).

[46] Sheppard, C. J. R., and Mao, X. Q., “Three-dimensional imaging in a microscope,” Journal of the Optical Society of America A 6(9), 1260-1269 (1989).

[47] Gu, M., [Advanced Optical Imaging Theory], Springer-Verlag, Berlin Heidelberg (2000).

[48] Wolf, E., "Three-dimensional structure determination of semi-transparent objects from holographic data," Optics Communications 1(4), 153-156 (1969).

[49] Mandal, R., Coupland, J., Leach, R. K., and Mansfield, D., "Coherence scanning interferometry: measurement and correction of three-dimensional transfer and point-spread characteristics," Applied Optics 53(8), 1554-1563 (2014).

[50] Su, R., Coupland, J. M., Sheppard, C. J. R., and Leach, R. K., "Scattering and three-dimensional imaging in surface-topography measuring interference microscopy," Journal of the Optical Society of America A 38(2), 27$42(2020)$.

[51] Coupland, J., Mandal, R., Palodhi, K., and Leach, R., "Coherence scanning interferometry: linear theory of surface measurement," Applied Optics 52(16), 3662-3670 (2013). 
[52] Coupland, J. M., and Lobera, J., "Holography, tomography and 3D microscopy as linear filtering operations," Measurement Science and Technology 19(7), 074012-1 - 074012-12 (2008).

[53] Su, R., Wang, Y., Coupland, J., and Leach, R. K., "On tilt and curvature dependent errors and the calibration of coherence scanning interferometry," Optics Express 25(4), 3297-3310 (2017).

[54] Su, R., Thomas, M., Leach, R. K., and Coupland, J., "Effects of defocus on the transfer function of coherence scanning interferometry," Optics Letters 43(1), 82-85 (2018).

[55] Su, R., Thomas, M., Liu, M., Drs, J., Bellouard, Y., Pruss, C., Coupland, J., and Leach, R., "Lens aberration compensation in interference microscopy," Optics and Lasers in Engineering 128, 106015 (2020). 